American Journal of Applied Sciences 5 (2): 152-157, 2008

ISSN 1546-9239

(C) 2008 Science Publications

\title{
Finite Element Analysis of Frictional Contact Problem During the Process of Metal Working
}

\author{
Nicolae Pop \\ North University of Baia Mare, Romania
}

\begin{abstract}
The quasistatic contact problem with dry friction, contributes to the achievement of a numerical model for the simultaneity of different kinds of viscoplastic, incompressible materials forming. Starting from a variational formulation of the problem, which extends the static case, a perturbed Lagrangean, discrete formulation within the framework of finite element method is obtained. On the contact boundary we have used a method based on contact finite elements, with three nodes, for the unilateral contact conditions with the friction law. One numerical example is presented.
\end{abstract}

Key words: Frictional contact problems, contact finite element, process of the metal working.

\section{INTRODUCTION}

The paper presents a formulation of the basic approach for finite element modeling of material forming processes with a viscoplastic, incompressible and frictional contact approximation.

Assume that the body is subject to volume forces while the input and output velocities are given and on a part of the boundary it is in a unilateral frictional contact with a rigid fixed support. Our interest is focused on the discretization by finite element of the contact area, such as the geometry of the contact area, the contact conditions and the frictional law is well approximated. The main numerical problems presented are: variational problem, evolving contact friction, modeling with finite contact element, incremental approach, meshing and remeshing of the domain, solving the non-linear set of equations using NewtonRaphson method.

The problem is also reduced to a set of optimization constrained problems. We solve this problem by using the Lagrange multipliers method. In order to illustrate the physical model the quasistatic problem is considered. The purpose is to find such velocities for the given boundary and constrained conditions that the forming processes are steady-state

\section{MATERIALS and METHODS}

Consider a Lipschitzian domain $\Omega$ on $R^{N}$, $N=1,2$, with boundary $\Gamma$. Let $\Gamma_{0}$ and $\Gamma_{1}$ be open and disjoint parts of $\Gamma$ so that $\Gamma=\Gamma_{0} \cup \Gamma_{1}$. We suppose that in the initial stage the body occupies the domain $\Omega$ and we restrict it to small displacement and small strains case.
Assume that the body is subjected to volume forces of density $\boldsymbol{f}=\left(f_{1}, f_{2}\right)$ on $\Omega$, while the velocities are given on $\Gamma_{0}$ with $m(\Gamma)>0$ and on $\Gamma_{1}$ it is in unilateral contact with a rigid fixed support. We use the following notations for the normal and tangential components of the velocities and of the stress vector:

$v_{n}=v_{i} n_{i}, \quad v_{t i}=v_{i}-v_{n} n_{i}, \quad \boldsymbol{\sigma}_{n}=\sigma_{i j} n_{i} n_{j}$, $\boldsymbol{\sigma}_{t}=\sigma_{i j} n_{j}-\sigma_{n} n_{i}$ where $i, j=1,2, \quad n=\left(n_{1}, n_{2}\right)$ are the outward normal unit vector on $\Gamma$ and the summation convention are used for $i$ and $j$.

The contact problem with friction law of elastoviscoplastic incompressible material model as the following classical formulation ${ }^{[1]}$ :

Find the field of velocities $v(x)=\left(v_{1}(x), v_{2}(x)\right)$, defined $\Omega$, which satisfy the following equations and conditions $\}$ :

the equilibrium equation

$$
\operatorname{div} \sigma+f=0 \text { on } \Omega
$$

- the incompressibility condition

$$
\operatorname{div} \boldsymbol{v}=0 \text { on } \Omega
$$

- the constitutive equation

$$
\boldsymbol{\sigma}^{D}=2 \eta \boldsymbol{D}_{I I} \boldsymbol{D}
$$

where $\begin{aligned} & \boldsymbol{\sigma}^{D}=\boldsymbol{\sigma}-\frac{\operatorname{tr} \boldsymbol{\sigma}}{3} I ; D_{i j}=\frac{1}{2}\left(\frac{\partial v_{i}}{\partial x_{j}}+\frac{\partial v_{j}}{\partial x_{i}}\right), \\ & D_{I I}=\frac{1}{2} D_{i j} D_{i j}, \sigma=\frac{1}{2} \sigma_{i j}^{D} \sigma_{i j}^{D} \text { and } \eta>0 \text { is }\end{aligned}$ viscosity coefficient. 
- the boundary conditions for field velocities:

$$
\begin{aligned}
& \boldsymbol{v}=v_{1} \text { on } \Gamma_{0}^{\prime} \\
& \boldsymbol{v}=v_{2} \text { on } \Gamma_{0}^{\prime \prime}
\end{aligned}
$$

- the unilateral contact conditions with friction law on $\Gamma_{1}=\Gamma_{1}^{\prime} \cup \Gamma_{1}^{\prime \prime}$ :

$v_{n} \leq 0$ with

$\boldsymbol{v}_{n}<0 \Rightarrow \boldsymbol{\sigma}_{n}=0$

$\boldsymbol{v}_{n}=0 \Rightarrow \boldsymbol{\sigma}_{n} \leq 0$ and

$\left\|\boldsymbol{\sigma}_{t}\right\|<\mu\left|\boldsymbol{\sigma}_{n}\right| \Rightarrow v_{t}=0$

$\left\|\boldsymbol{\sigma}_{t}\right\|<\mu\left|\boldsymbol{\sigma}_{n}\right| \Rightarrow \exists \lambda \geq 0$

with $\boldsymbol{\sigma}_{t}=-\lambda \boldsymbol{v}_{t}$ follows:

The classical formulation of this problem is as

Problem P1: Find the velocities $v=\left(v_{1}, v_{2}\right)$, which satisfy (1) - (6), where $\boldsymbol{f}, v_{1}, v_{2}, \mu$ and $\eta$ are given.

It is known ${ }^{[2]}$ that a variational formulation of the Problem P1 is the following inequality:

Problem P2: Find $\boldsymbol{v}=\left(v_{1}, v_{2}\right)$ such that

$a(v, u-v)+\frac{1}{\varepsilon} \int \operatorname{div} v \operatorname{div}(u-v) d x+$

$+J(v, u)-J(v, v)>(L, u-v), \forall u \in K$,

where

$K=\left\{u \in\left(H^{1}(\Omega)\right)^{N}\right.$,

$u=u^{i}$ a.i. on $\Gamma_{0}^{\prime}, u=u^{e}$ a.i. on $\Gamma_{0}^{\prime \prime}$,

$\operatorname{div} u=0$ on $\Omega$, and $u_{n} \leq 0$ a.e. on $\left.\Gamma^{1}\right\}$,

$a(v, u)=2 \int_{\Omega} \eta\left(D_{I I}(v)\right) D_{i j}(v) D_{i j}(u) d x$,

$\forall u, v \in\left(H^{1}(\Omega)\right)^{N}$,

$J(u, v)=\int_{\Gamma_{1}} \mu\left|\sigma_{n}(v)\right|\left\|u_{t}\right\| d s$,

$L(u)=\int_{\Omega} f_{i} u_{i} d x, \forall u \in\left(H^{1}(\Omega)\right)^{N}$,

where $N=1,2 ; f=\left(f_{1}, f_{2}\right) \in\left(H^{1}(\Omega)\right)^{N}, \quad \mathcal{E}$ is the penalty parameter of the unsatisfactory of the incompressibility condition.

Finite element discretization of the problem: We consider a discrete variational problem
$P 2$ using four nodes isoparametric finite elements on $\Omega$, and three nodes contact element ${ }^{[3,4]}$ on $\Gamma_{1}$.

The contact finite elements with 3 nodes (see Figure 1) is a bilinear isoparametric element which associates one gap with a typical slave node $S$ is given by $g=\left(x_{s}-x_{1}\right) n$, where $n$ denotes the normal master segment 1-2, $x_{s}=X_{s}+v_{s}$ defines the current position of the slave node, and $x_{1}=X_{1}+v_{1}$, $x_{2}=X_{2}+v_{2}$ define the current position of the master segment.

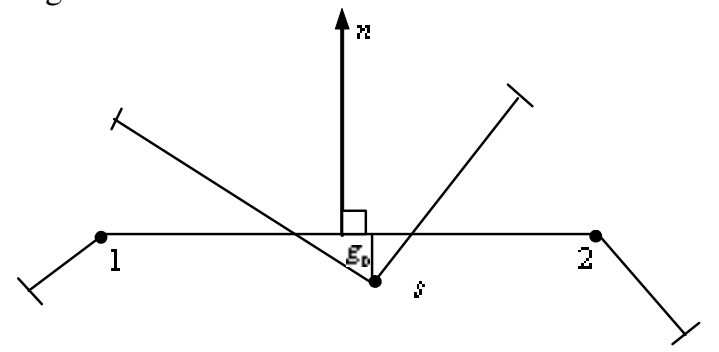

Fig. 1: Geometry of a slave node with a master segment 1-2

In all numerical applications we have derived a perturbed Langrangean formulation for the case of frictional stick and for the case of frictional slide. For the case of frictional stick the perturbed Lagrangean functional for bodies in contact has the following form:

$L\left(v, \Sigma_{n}, \Sigma_{t}, D_{v}\right)=\prod(v)+\sum_{n}^{T} G_{n}+$

$+\sum_{n}^{T} G_{t}-\frac{1}{2 \omega_{n}} \sum_{n}^{T} \Sigma_{n}-\frac{1}{2 \omega_{t}} \sum_{t}^{T} \Sigma_{t}+$

$+\frac{1}{2 \varepsilon} D_{v}^{T} D_{v}$

where $v$ is the vector of nodal velocities $\Sigma_{n}, \Sigma_{t}$, are the vectors of normal and tangential nodal contact forces, respectively, $G_{n}$ and $G_{t}$ are the vectors of normal and tangential nodal gaps, and $\omega_{n}, \omega_{t}$ are the normal and tangential penalty parameters respectively, $\prod(v)$ are the total potential energy, $\mathcal{E}$ is the penalty parameter of the unsatisfaction of the incompressibility condition and $D_{v}$ is the vector obtained from the incompressibility condition.

For the case of frictional slide the relation $\left\|\sum_{t}\right\|=\mu\left\|\sum_{n}\right\|$ must be considered, as a direct consequence of the Coulomb's friction law. 
The Newton-Raphson method was applied to the discrete variational formulations that can be derived from these perturbed Lagrangean functional.

In the three dimensional case $^{[7]}$, a four node contact element consisting of three "master" nodes 1, 2, 3 and one "slave" node's, is employed (see Figure 2).

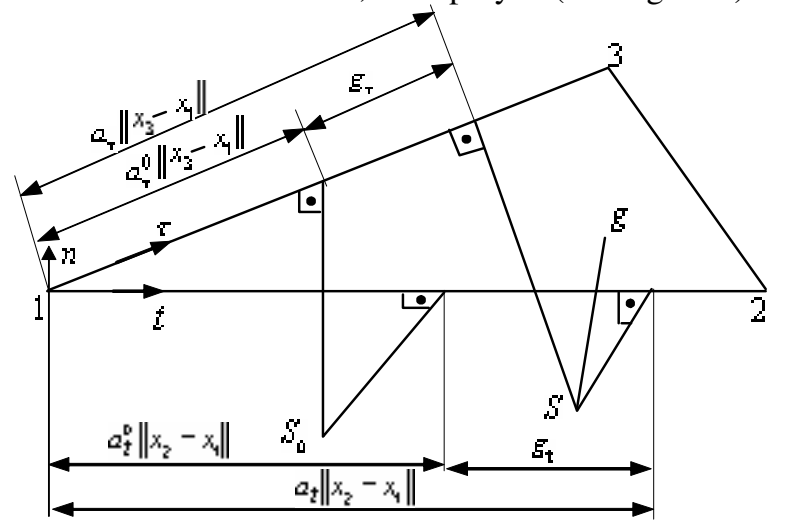

Fig. 2: Three-dimensional contact finite element

Normal vectors on a defined plane by the nodes 1 , 2 and 3 and respectively the vectors, defined by directions of the nodes 1-2 and 1-3 will be:

$$
\begin{aligned}
& n=\frac{\left(x_{2}-x_{1}\right) \cdot\left(x_{3}-x_{1}\right)}{\left\|\left(x_{2}-x_{1}\right) \cdot\left(x_{3}-x_{1}\right)\right\|} ; t=\frac{x_{2}-x_{1}}{\left\|x_{2}-x_{1}\right\|} ; \\
& \tau=\frac{x_{3}-x_{1}}{\left\|x_{3}-x_{1}\right\|}
\end{aligned}
$$

where $x_{1}=X_{1}+u_{1}, \quad x_{2}=X_{2}+u_{2}, \quad x_{3}=X_{3}+u_{3}$ signify the current positions of master nodes; $X_{1}, X_{2}, X_{3}$ are reference coordinates and $u_{1}, u_{2}, u_{3}$ are current nodal displacements of points 1,2 and 3 .

In addition, we define the current "surface coordinate" as following:

$$
a_{t}=\frac{\left(x_{s}-x_{1}\right)}{\left\|x_{2}-x_{1}\right\|} t ; a_{\tau}=\frac{\left(x_{s}-x_{1}\right)}{\left\|x_{2}-x_{1}\right\|} \tau
$$

where $x_{s}=X_{s}+u_{s}$ denotes the current position of the slave node. The normal and tangential gaps $g_{n}, g_{t}$, $g_{\tau}$ are defined as

$$
\begin{aligned}
& g_{n}=\left(x_{s}-x_{1}\right) n, \\
& g_{t}=\left(a_{t}-a_{t}^{0}\right)\left\|x_{2}-x_{1}\right\|, \\
& g_{\tau}=\left(a_{\tau}-a_{\tau}^{0}\right)\left\|x_{3}-x_{1}\right\|,
\end{aligned}
$$

where $a_{t}^{0}$ and $a_{\tau}^{0}$ are the old surface coordinates at the last time step known.
Note that the gap $g$ depends on the slave node $s$ as well as on the master nodes 1,2 and 3 . Thus, the variation of the gap is obtained according to

$$
\begin{gathered}
g=\frac{d}{d \alpha}\left[g \left(x_{s}+\alpha \eta_{s}, x_{1}+\alpha \eta_{1}, x_{2}+\alpha \eta_{2},\right.\right. \\
\left.x_{3}+\alpha \eta_{3}\right]
\end{gathered}
$$

where

$$
\eta\left(\eta_{1}, \eta_{2}, \eta_{3}, \eta_{s}\right) \equiv \delta u\left(\delta u_{1}, \delta u_{2}, \delta u_{3}, \delta u_{s}\right)
$$

element implementations, explicit matrix expressions for the Lagrangean multiplier formulation and the penalty formulation are derived as follows.

The discrete variational equation associated with (12) take the form:

$$
\begin{aligned}
& \delta_{u} \Pi(u)+\Sigma_{n}^{T} \delta_{u} G_{n}+\Sigma_{t}^{T} \delta_{u} G_{t}+\Sigma_{\tau}^{T} \delta_{u} G_{\tau}=0 \\
& \delta \Sigma_{n}^{T}\left(-\frac{1}{\omega_{n}} \Sigma_{n}+G_{n}\right)=0 \\
& \delta \Sigma_{t}^{T}\left(-\frac{1}{\omega_{t}} \Sigma_{t}+G_{t}\right)=0 \\
& \delta \Sigma_{\tau}^{T}\left(-\frac{1}{\omega_{\tau}} \Sigma_{\tau}+G_{\tau}\right)=0,
\end{aligned}
$$

where $\Pi(u)=\frac{1}{2} a(u, u)-L(u)$ is the total potential energy of the bodies in contact,

$\delta_{u} G_{n}=\left(\delta_{u} g_{n}^{1}, \delta_{u} g_{n}^{2}, \ldots, \delta_{u} g_{n}^{s}\right)^{T}$,

$\delta_{u} G_{t}=\left(\delta_{u} g_{t}^{1}, \delta_{u} g_{t}^{2}, \ldots, \delta_{u} g_{t}^{s}\right)^{T}$,

$\delta_{u} G_{\tau}=\left(\delta_{u} g_{\tau}^{1}, \delta_{u} g_{\tau}^{2}, \ldots, \delta_{u} g_{\tau}^{s}\right)^{T}, S=$ total number of slave nodes in contact $s=1,2, \ldots, S$, analogous for $\delta \Sigma_{n}$, $\delta \Sigma_{t}, \delta \Sigma_{\tau}$.

The variational of a typical nodal normal gap $g_{n} \in G_{n}$ take the form:

$\delta g_{n}=\sum_{j=1}^{3} \frac{\partial g_{n}}{\partial u_{s}^{j}} \eta_{s}^{j}+\sum_{i=1}^{3} \sum_{j=1}^{3} \frac{\partial g_{n}}{\partial u_{i}^{j}} \eta_{i}^{j}, \quad$ with the notation (13) and

$c_{n}=\left(\frac{\partial g_{n}}{\partial u_{s}^{1}}, \frac{\partial g_{n}}{\partial u_{s}^{2}}, \frac{\partial g_{n}}{\partial u_{s}^{3}}, \frac{\partial g_{n}}{\partial u_{1}^{1}}, \ldots, \frac{\partial g_{n}}{\partial u_{3}^{3}}\right)$,

$\eta=\left(\eta_{s}^{1}, \eta_{s}^{2}, \eta_{s}^{3}, \eta_{1}^{1}, \ldots, \eta_{3}^{3}\right)$, we obtain:

$\delta g_{n}=\eta^{T} c_{n}$. 
Similarly, the variation of a typical nodal tangential gap $g_{t} \in G_{t}, \quad g_{\tau} \in G_{\tau}$ can be obtained according to $\delta g_{t}=\eta^{T} c_{t}, \quad \delta g_{\tau}=\eta^{T} c_{\tau}$.

Moreover, the residual vector $R_{B}$ and the tangent stiffness $K_{B}$ associated, with the total potential energy of the contacting bodies simply read, result

$$
\delta \Pi(u)=\eta^{T} R_{B} \quad \text { and } \quad \delta R_{B}=\eta^{T} K_{B} .
$$

With, the convention:

$$
\begin{gathered}
\left(u^{1}, \ldots, u^{12}\right)=\left(u_{s}^{1}, u_{s}^{2}, u_{s}^{3}, u_{1}^{1}, \ldots, u_{3}^{3}\right) \quad \text { (14) become: } \\
\eta^{T}\left[R_{B}+\sum_{s=1}^{S}\left(\sigma_{n}^{s} c_{n}^{s}+\sigma_{t}^{s} c_{t}^{s}+\sigma_{\tau}^{s} c_{\tau}^{s}\right)\right]=0
\end{gathered}
$$

and analogously for (15)-(17) where

$\sigma_{n} \in \Sigma_{n}, \sigma_{t} \in \Sigma_{t}, \sigma_{\tau} \in \Sigma_{\tau}$.

To apply Newton's iteration scheme, consistent linearization of (18) and those corresponding (15)(17), at $\left(u, \Sigma_{u}, \Sigma_{t}, \Sigma_{\tau}\right)$ is performed and leads

$\left[\eta^{T}, \delta \Sigma_{n}^{t}, \delta \Sigma_{t}^{T}, \delta \Sigma_{\tau}^{T}\right]\left\{\left[\begin{array}{cccc}A_{1} & A_{2} & A_{3} & A_{4} \\ A_{2}^{T} & B_{2} & O & O \\ A_{3}^{T} & O & C_{3} & O \\ A_{4}^{T} & O & O & D_{4}\end{array}\right]\left\{\begin{array}{l}\Delta u \\ \Delta \Sigma_{n} \\ \Delta \Sigma_{t} \\ \Delta \Sigma_{\tau}\end{array}\right\}=-\left\{\begin{array}{l}R_{1} \\ R_{2} \\ R_{3} \\ R_{4}\end{array}\right\}\right\}$

where

$$
\begin{aligned}
& A_{1}=K_{B}+\sum_{s=1}^{S}\left(k_{n}^{s}+k_{t}^{s}+k_{\tau}^{s}\right), A_{2}=\sum_{s=1}^{S} c_{n}^{s}, A_{3}=\sum_{s=1}^{S} c_{t}^{s}, \\
& A_{4}=\sum_{s=1}^{S} c_{\tau}^{s}, B_{2}=-\frac{1}{\omega_{n}} I, C_{3}=-\frac{1}{\omega_{t}} I, D_{4}=-\frac{1}{\omega \tau} I, \\
& R_{1}=R_{B}+\sum_{s=1}^{S}\left(\sigma_{n}^{s} c_{n}^{s}+\sigma_{t}^{s} c_{t}^{s}+\sigma_{\tau}^{s} c_{\tau}^{s}\right) \\
& R_{2}=-\frac{1}{\omega_{n}} \Sigma_{n}+G_{n}, R_{3}=-\frac{1}{\omega_{t}} \Sigma_{t}+G_{t}, \\
& R_{4}=-\frac{1}{\omega_{\tau}} \Sigma_{\tau}+G_{\tau},
\end{aligned}
$$

$\mathrm{O}$ is the matrix zero, and

$$
\begin{aligned}
\left(k_{n}^{s}\right)_{j i} & =\frac{\partial c_{n}^{s i}}{\partial u_{j}}=\frac{\partial^{2} g_{n}^{s}}{\partial u_{i} \partial u_{j}},\left(k_{t}^{s}\right)_{j i}=\frac{\partial c_{t}^{s i}}{\partial u_{j}}=\frac{\partial^{2} g_{t}^{s}}{\partial u_{i} \partial u_{j}} \\
\left(k_{\tau}^{s}\right)_{j i} & =\frac{\partial c_{\tau}^{s i}}{\partial u_{j}}=\frac{\partial^{2} g_{\tau}^{s}}{\partial u_{i} \partial u_{j}} .
\end{aligned}
$$

Finally after the discrete formulation within the framework FEM, a standard assembly procedure can be used to add the contact contributions of each contact element to the global tangent stiffness and residual matrix and thus we obtain:

$$
K U=R \text {, }
$$

where $K=K_{B}+\sum_{s=1}^{S} K_{C}^{s}, R=-\left(R_{B}+\sum_{s=1}^{S} R_{C}^{s}\right)$,

$K_{B}, R_{B}$ are mechanical global tangent stiffness matrix and residual vector, $K_{C}^{s}, R_{C}^{s}$ are mechanical contact contributions of contact nod $S$, $U=\left(\Delta u, \Delta \Sigma_{n}, \Delta \Sigma_{t}, \Delta \Sigma_{\tau}\right)^{T}, S$ is the total number of the slave nodes. And for $\omega_{n}=\omega_{t}=\omega_{\tau}=\omega$, and $\sigma_{n}=\omega g_{n}, \sigma_{t}=\omega g_{t}, \sigma_{\tau}=\omega g_{\tau}$ result $K_{C}=$

$$
\begin{aligned}
& =\sum_{s=1}^{S} \omega\left(g_{n}^{s} k_{n}^{s}+g_{t}^{s} k_{t}^{s}+g_{n}^{s} k_{\tau}^{s}+c_{n}^{s T} c_{n}^{s}+c_{t}^{s T} c_{t}^{s}+c_{\tau}^{s T} c_{\tau}^{s}\right) \\
& R_{C}=\sum_{s=1}^{S} \omega\left(g_{n}^{s T} c_{n}^{s}+g_{t}^{s T} c_{t}^{s}+g_{\tau}^{s T} c_{\tau}^{s}\right)
\end{aligned}
$$

For the case of frictional slide we used the relation $\left|\Sigma_{\tan }\right|=\mu\left|\Sigma_{n}\right|$, where $\mu$ is the coefficient of friction and $\Sigma_{\tan }$ is the result force of the $\Sigma_{t}$ and $\Sigma_{\tau}$, forces in the tangent plane of the contact surface.

Note with $\beta$ the angle between the sides $\overline{x_{2}-x_{1}}$ and $\overline{x_{3}-x_{1}}$; we obtain $\cos \beta=t \tau$ and $\left|\lambda_{\tan }\right|=\mu \sqrt{g_{t}^{2}+g_{\tau}^{2}+2 \varepsilon\left|g_{t}\right|\left|g_{\tau}\right| \cos \beta}$ where $\varepsilon=\operatorname{sgn}\left(g_{t} g_{\tau}\right)$. As a direct consequence of Coulomb's friction law, it results $\mu \omega\left|g_{n}\right|=\omega r$, where $r=\sqrt{g_{t}^{2}+g_{\tau}^{2}+2 \varepsilon\left|g_{t}\right|\left|g_{\tau}\right| \cos \beta}$ therefore $\lambda_{t}=\lambda \tan \frac{g_{t}}{r} \omega g_{n}=-\mu \operatorname{sgn}\left(g_{t}\right) \frac{g_{t}}{r} \omega g_{n}=-\mu \frac{\left|g_{t}\right|}{r} \omega g_{n}$, $\lambda_{\tau}=-\mu \frac{\left|g_{\tau}\right|}{r} \omega g_{n}$.

If we write $d_{t}=\frac{\left|g_{t}\right|}{r}, d_{\tau}=\frac{\left|g_{\tau}\right|}{r}, b_{t}=\frac{\partial d_{t}}{\partial u}, b_{\tau}=\frac{\partial d_{\tau}}{\partial u}$, from linearized kinematics (i.e., by neglecting nonlinear terms $k_{t}$ and $k_{\tau}$ ), we obtain: $K_{C}=\sum_{s=1}^{S}\left(S L_{1}^{s}+S L_{2}^{S}\right)$, with 


$$
\begin{aligned}
& S L_{1}^{S}=\omega\left(g_{n}^{s} k_{n}^{s}-\mu g_{n} d_{t}^{s} k_{t}^{s}-\mu g_{n}^{s} d_{\tau}^{s} k_{\tau}^{s}+c_{n}^{s T} c_{n}^{s}-\right. \\
& \left.-\mu d_{t}^{s} c_{n}^{s T} c_{t}^{s}\right) \\
& S L_{2}^{S}=\omega\left(-\mu d_{t}^{s} c_{n}^{s T} c_{\tau}^{s}-\mu g_{n} b_{t}^{s T} c_{t}^{s}-\mu g_{n} b_{\tau}^{s T} c_{\tau}^{s}\right) \text { and } \\
& R_{c}=\sum_{s=1}^{s} \omega\left(\mu g_{n} d_{t}^{s T} c_{t}^{s}+\mu g_{n} d_{\tau}^{s T} c_{\tau}^{s}-g_{n}^{s T} c_{n}^{s}\right) .
\end{aligned}
$$

The Newton-Raphson method was applied to the discrete variational formulation that can be derived from these perturbed Lagrangian functional.

The scheme of solving the linearized problem is following:

(i) initialisation set the iterative count $k=0$, $u^{(0)}=0$;

(ii) compute the system stiffness and residual excluding contact;

(iii) compute contact stiffness and residual for each finite element $s, s=1, \ldots, N C$

a) compute the normal gap $g_{n}^{(s)(k)}$;

b) check for contact finite element status:

IF $g_{n}^{(s)(k)}>$ TOL then out of contact

ELSE in contact. Check for frictional stick or slip; compute $\sum_{n}^{(k)}, \sum_{t}^{(k)}, \sum_{\tau}^{(k)}$ and

$\operatorname{IF}\left|\operatorname{SQRT}\left(\left(\sum_{t}^{(k)}\right)^{2}+\left(\sum_{\tau}^{(k)}\right)^{2}\right)\right| \leq \mu\left|\sum_{n}^{(k)}\right|$ then

frictional stick;

\section{ENDIF}

ELSE frictional slip; ENDIF

c) compute the total stiffness and residual;

(iv) solve the total system to obtain displacement increment $\Delta u^{(k)}$;

(v) check for convergence:

IF $\left|\Delta u^{(k)}-\Delta u^{(k+1)}\right|<$ TOL1 then converge and exit.

ELSE go to step (vi);

(vi) update the displacement field $u^{(k+1)}=u^{(k)}+\Delta u^{(k+1)}$;

(vii) set $k=k+1$ and go to back to (ii).

Several examples have been taken from other works to be compared and the results obtained by us agreed to the ones ${ }^{[7]}$.

\section{RESULTS and DISCUSSION}

Based on these matrices, a standard assembly procedure can be used to add the contact contributions of each contact node to the global tangent stiffness and residual.

We $\operatorname{consider}^{[5,6]}$ a simplified model with the geometry and loading given in Figure 3, a discretization with 32 nodes and 20 finite elements with four nodes isoparametric and for contact boundary, three nodes contact element ${ }^{[4]}$. Data of the problem:

$h_{i}=38 \mathrm{~mm}$;

$h_{0}=9,5 \mathrm{~mm}$;

$\mu=0,001$ on $\Gamma_{1}^{\prime}$ and $\mu=0,1$ on $\Gamma_{1}^{\prime \prime}$;

$\varepsilon=10^{-7} \eta_{m}$, with $\eta_{m}$ in average value of $\eta$, and $\eta_{0}=0,278$ Mpas;

$\beta=70^{\circ}, v_{i}=0,19 \mathrm{~m} / \mathrm{s}$, where we have used index $i$ for input data and index $o$ for output data.

Obtained values for $v_{1}$ and $\left\|v_{2}\right\|$, where $\boldsymbol{v}=\left(v_{1}, v_{2}\right)$, are represented in Figure 4, respective Figure 5.

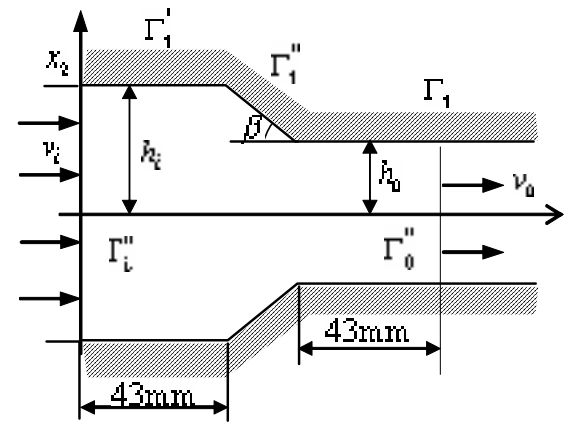

Fig. 3: Geometry of domain $\Omega$

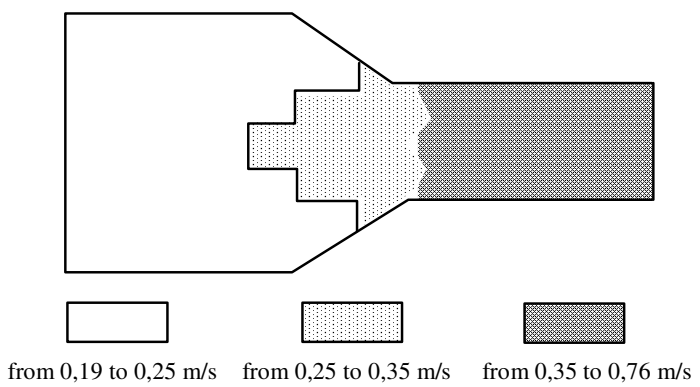

Fig. 4: Values of the $v_{1}$ 


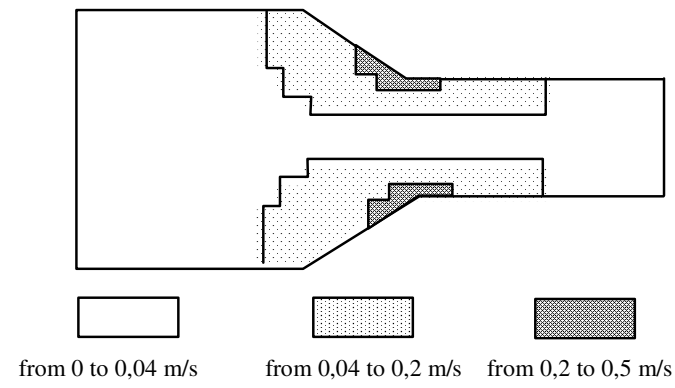

Fig. 5: Values of the $\left\|v_{2}\right\|$

\section{CONCLUSION}

This study investigates the simulation model of different kinds of viscoplastic, incompressible materials forming. Starting with the initial value $\eta_{0}=0,278$ MPas for viscosity coefficient, a fast convergence of the sequence (7) in the discretization variant had been observed. Only three iterations are enough to obtain a relative error less than one percent. The problem can be extended in the three-dimensional case and for crack analysis ${ }^{[8]}$.

\section{ACKNOWLEDGEMENT}

The author acknowledgements support from Ministry of education and Research under CEEX Grant No. 2-CEEX-06-11-96/19.09.2006.

\section{REFERENCES}

1. Teodosiu, C., Soós, E. and Rosu, T., 1990. A finite element model of the hot working of axisymmetric products, Rev. Roum., Sci., Tech., Mec. Appl., III , 35: 225-229
2. Klarbring, A., Mikelic, A., Shillor, M., 1988. Frictional Contact Problems with Normal Compliance, Int. J. Eng. Sci. 26 : 811-832Ju, J. W. and Taylor, R. L., 1988. A Perturbed Lagrangean Formulation for the Finite Element Solution of Nonlinear Contact Problems, J. de Méc. Théor. et App., Special Issue, Supplement no. 1 to vol. 7:114.

3. Wriggers, P. and Simo, J. C., 1985. A Note on Tangent Stiffness for Frictional Contact Problems, Comm. in App. Num. Meth., 1: 199-203.

4. Cocu, M., 1991. Nonlinear boundary value problems in the termo-viscoplasticity of the metals (in Romanian), Ph Thesis, Bucharest University.

5. Rous, M., Chabrand, P. and Lebon, F., 1988. Numerical Methods for Frictional Contact Problems and Applications, J. de Méc. Théor. et App., Special Issue, Supplement no. 1 to vol. 7 : 111-126

6. Pop, N., 2004. Numerical Simulations for the 3D Frictional Contact Problems, Inginerare, Revista Falculdad de Ingenieria de la Pontificia Universidad Catolica de Valparaiso, CHILE, 17: 33-38

7. Labibzadeh, M., Sadrnejad, S.A., 2007. Crack Analysis of Concrete Arch Dams Using MicroPlanes Damage Based Constitutive Relations, American Journal of Applied Sciences, 4: 197202. 